\title{
Mapeamento do saldo de radiação com imagens Landsat 5 e modelo de elevação digital ${ }^{1}$
}

\author{
Frederico T. Di Pace ${ }^{2}$, Bernardo B. da Silva ${ }^{3}$, Vicente de P. R. da Silva ${ }^{3}$ \& Saulo T. A. da Silva ${ }^{4}$
}

\begin{abstract}
RESUMO
O saldo de radiação é um importante componente do balanço de energia e tem grande relevância em estudos de evapotranspiração em áreas irrigadas e em bacias hidrográficas. Obteve-se, através do estudo, a estimativa do saldo de radiação à superfície terrestre, mediante imagens multiespectrais do Mapeador Temático do satélite Landsat 5, utilizando-se o SEBAL (Surface Energy Balance Algorithm for Land) e o MED (Modelo de Elevação Digital). Os cálculos foram realizados com e sem utilização do MED, nos dias 04 de dezembro de 2000 e 04 de outubro de 2001. A temperatura da superfície (Ts) e os valores do albedo estimados com o MED em 04/12/2000, foram um pouco superiores aos valores de Ts estimados sem a utilização deste modelo. Os resultados demonstraram que na estimativa do saldo de radiação com base em imagens MT - Landsat 5, se deve levar em consideração os efeitos topográficos da região de estudo.
\end{abstract}

Palavras-chave: radiância, albedo da superfície, emissividade

\section{Mapping net radiation using Landsat 5 imagery and digital elevation model}

\begin{abstract}
Net radiation is an important component of the surface energy balance in studies of evapotranspiration of irrigated crops and in evaporation of hydrological basins. The objective of this research was to determine the surface radiation balance, by using multispectral imagery of the Thematic Mapper (Landsat 5 satellite). In this study the SEBAL (Surface Energy Balance Algorithm for Land) and DEM (Digital Elevation Model) were used in order to correct the albedo and vegetation indices under the influence of the slope aspects were used for each study area. TM (Thematic Mapper) imageries were used for two different dates (December 4, 2000 and October 4, 2001). The calculations were accomplished with and without use of the DEM. The land surface temperature and albedo values with DEM were larger than without DEM in both years, for two selected areas. Results also show that for obtaining net radiation based on imagery of the TM Landsat 5 the topographical effects of the study area must be considered.
\end{abstract}

Key words: radiance, surface albedo, emissivity

\footnotetext{
1 Parte da Tese do primeiro autor apresentada ao Programa de Pós-Graduação em Recursos Naturais

${ }^{2}$ ICA/UFAL. E-mail: fred@ccen.ufal.br

3 UACA/UFCG. Av. Aprígio Veloso 822. Bodocongó, CEP 58109-970, Campina Grande, PB. E-mail: bernardo@dca.ufcg.edu.br; vicente@dca.ufcg.edu.br

${ }^{4}$ Doutorando do Programa de Pós-Graduação em Meteorologia/UFCG
} 


\section{INTRODUÇÃO}

O conhecimento do saldo de radiação é fundamental em estudos agrometeorológicos, de monitoramento climático e previsão de tempo. Muitos instrumentos são capazes de medir com boa precisão esse importante componente do balanço de energia; entretanto, aqueles considerados convencionais proporcionam medidas pontuais do saldo de radiação que são representativas apenas para áreas de pequena dimensão. Quando há necessidade de estimativa desse elemento climático em grandes áreas, as técnicas baseadas em medições realizadas por sensores orbitais são mais apropriadas porque reúnem grande cobertura espacial e têm baixo custo operacional.

Nos últimos anos têm sido propostos alguns algoritmos para o cálculo do balanço de energia à superfície; o mais destacado é o SEBAL (Surface Energy Balance Algorithm for Land), pois leva em consideração a variabilidade espacial de muitos elementos micrometeorológicos, através de funções semi-empíricas e pode ser aplicado em diversos agrossistemas (Bastiaanssen et al., 1998); este algoritmo é capaz de realizar estimativas precisas da evapotranspiração de áreas agrícolas relativamente horizontais. Porém, nos casos em que a topografia da área não é plana nem horizontal, pode haver considerável comprometimento na estimativa dos componentes do balanço de radiação e, conseqüentemente, na estimativa da evapotranspiração de culturas agrícolas e/ou de vegetação natural; a razão disso é que o ângulo zenital do Sol, relativo à normal de cada pixel, difere daquele relacionado a uma superfície horizontal e, como resultado, pode haver erros apreciáveis no cálculo da refletividade monocromática e, consequentemente, nos demais parâmetros dela derivados. O SEBAL tem sido utilizado em diferentes países e produzido resultados significativos, com vistas à racionalização do uso dos recursos naturais, avaliação de grandes áreas irrigadas, manejo da irrigação e gerenciamento de recursos hídricos (Morse et al., 2000; Bastiaanssen, 2000; Granger, 2000; Silva et al., 2005); no entanto, ainda persistem algumas questões relacionadas aos efeitos, ou seja, da irregularidade que a topografia do terreno provoca no cômputo do albedo superficial e, conseqüentemente, no saldo de radiação.

Ante o exposto se objetiva, neste trabalho, estimar o saldo de radiação à superfície terrestre mediante dados de imagens multiespectrais do satélite Landsat 5, considerandose a topografia da superfície com a correção do albedo superficial.

\section{MATERIAL E MÉTODOS}

A região de estudo foi o Perímetro Irrigado Senador Nilo Coelho e áreas de vegetação nativa adjacentes. Esta região compreende a margem esquerda do Rio São Francisco, estendendo-se da Barragem de Sobradinho, localizada no município de Casa Nova, BA, até o município de Petrolina, PE, totalizando, assim, uma área de 23.000 ha.

Utilizaram-se, para o estudo, duas imagens obtidas pelo satélite Landsat 5, compostas de sete bandas espectrais, cujas características são apresentadas na Tabela 1; essas imagens correspondem à passagem do TM - Landsat 5 sobre a área de interesse, às 9 h 30 min (tempo solar) dos dias 04 de dezembro de 2000 e 04 de outubro de 2001, na órbita 217 e ponto 67.

Na Figura 1 é apresentada a imagem da área de estudo, em composição RGB (red, green and blue) das bandas 2, 3 e 4 do TM - Landsat 5, recortada com o software ERDAS Imagine 8.5. Dois alvos foram analisados e a eles se deram as seguintes denominações: alvo P1(área irrigada) e alvo P2 (solo exposto). Os valores dos coeficientes de calibração radiométrica do TM - Landsat 5, são aqueles sugeridos por Allen et al. (2002) para imagens obtidas a partir do ano 2000; o alvo P1 apresentava inclinação de 3 graus, que no alvo P2 era de 7 graus, ambos direcionados para o nordeste.

Tabela 1. Descrição das bandas do Mapeador Temático (TM) do Landsat 5, com os correspondentes intervalos de comprimentos de onda, coeficientes de calibração (radiância mínima - a e máxima - b) e irradiância solar espectral no topo da atmosfera $\left(\mathrm{K}_{\lambda i}\right)$

\begin{tabular}{|c|c|c|c|c|}
\hline \multirow{2}{*}{$\begin{array}{l}\text { Descrição } \\
\text { das bandas* }\end{array}$} & \multirow{2}{*}{$\begin{array}{l}\text { Comprimento } \\
\text { de onda }(\mu \mathrm{m})\end{array}$} & \multicolumn{2}{|c|}{$\begin{array}{l}\text { Coeficientes de calibração } \\
\qquad\left(W \mathrm{~m}^{-2} s r^{-1} \mu \mathrm{m}^{-1}\right)\end{array}$} & \multirow{2}{*}{$\begin{array}{c}\mathrm{K} \text { I } \\
\left(\mathrm{W} \mathrm{m}^{-2} \mu \mathrm{m}^{-1}\right.\end{array}$} \\
\hline & & a & b & \\
\hline Banda 1 (Azul) & $0,45-0,52$ & $-1,765$ & 178,941 & 1957 \\
\hline Banda 2 (Verde) & $0,52-0,60$ & $-3,576$ & 379,055 & 1829 \\
\hline Banda 3 (Vermelho) & $0,63-0,69$ & $-1,502$ & 255,695 & 1557 \\
\hline Banda 4 (IV próximo) & $0,76-0,90$ & $-1,763$ & 242,303 & 1047 \\
\hline Banda 5 (IV médio) & $1,55-1,75$ & $-0,411$ & 30,178 & 219,3 \\
\hline Banda 6 (IV termal) & $10,4-12,5$ & 1,238 & 15,600 & - \\
\hline Banda 7 (IV médio) & $2,08-2,35$ & $-0,137$ & 13,156 & 74,52 \\
\hline
\end{tabular}

No estudo se utilizou o Modelo de Elevação Digital (MED) com a mesma resolução espacial e recorte das imagens TM Landsat 5. A partir do MED foram criadas imagens da elevação (m), declividade (\%) e ângulo azimutal (graus) da normal de cada pixel. Na Tabela 2 constam os valores médios (mensal e anual) de algumas variáveis meteorológicas da estação agrometeorológica de Bebedouro, Petrolina, PE, enquanto se apresentam, nas subseções seguintes, as etapas de processamento das imagens para obtenção do saldo de radiação.

\section{Calibração radiométrica}

A radiância espectral de cada banda $\left(\mathrm{L}_{\lambda \mathrm{i}}\right)$ representa a energia solar refletida por cada pixel por unidade de área, de tempo, de ângulo sólido e de comprimento de onda, medida a nível do satélite Landsat (aproximadamente $705 \mathrm{~km}$ de altura), para as bandas 1, 2, 3, 4, 5 e 7; para a banda 6, essa radiância representa a energia emitida por cada pixel e pode ser obtida pela equação (Markham \& Baker, 1987):

$$
\mathrm{L}_{\lambda_{\mathrm{i}}}=\mathrm{a}_{\mathrm{i}}+\frac{\mathrm{b}_{\mathrm{i}}-\mathrm{a}_{\mathrm{i}}}{255} \mathrm{ND}
$$

em que a e b são as radiâncias espectrais mínima e máxima $\left(\mathrm{W} \mathrm{m}^{-2} \mathrm{sr}^{-1} \mu \mathrm{m}^{-1}\right)$, detectadas pelo $\mathrm{TM}$ - Landsat 5, 
Tabela 2. Valores médios mensais e anuais de algumas variáveis meteorológicas da estação agrometeorológica de Bebedouro, Petrolina, PE $\left(09^{\circ} 09^{\prime} \mathrm{S} ; 40^{\circ} 22^{\prime} \mathrm{W} ; 315,9 \mathrm{~m}\right)$. Os valores da precipitação pluvial estão expressos como totais mensais e total anual (mm)

\begin{tabular}{|c|c|c|c|c|c|c|c|c|c|c|c|c|c|}
\hline Ano & Jan & Fev & Mar & Abr & Mai & Jun & Jul & Ago & Set & Out & Nov & Dez & Média \\
\hline \multicolumn{14}{|c|}{ Temperatura do ar $\left({ }^{\circ} \mathrm{C}\right)$} \\
\hline 2001 & 26,1 & 26,5 & 25,7 & 25,5 & 26,1 & 23,8 & 23,7 & 23,6 & 25,7 & 27,3 & 28,1 & 27,7 & 25,1 \\
\hline 2000 & 25,1 & 24,8 & 25,1 & 25,1 & 24,8 & 23,7 & 23,4 & 24,7 & 25,7 & 26,9 & 25,8 & 25,5 & 25,0 \\
\hline \multicolumn{14}{|c|}{ Precipitação pluvial (mm) } \\
\hline 2001 & 4,8 & 61,5 & 209,6 & 16 & 0,6 & 35,6 & 4,9 & 6,9 & 2,0 & 0,6 & 1,0 & 75,9 & 403,4 \\
\hline 2000 & 69,9 & 78,8 & 81,6 & 92,6 & 21,9 & 15,0 & 3,8 & 1,4 & 1,0 & 1,9 & 144,6 & 129,7 & 642,2 \\
\hline \multicolumn{14}{|c|}{ Umidade relativa (\%) } \\
\hline 2001 & 61 & 67 & 72 & 68 & 63 & 69 & 67 & 64 & 60 & 56 & 57 & 61 & 64 \\
\hline 2000 & 83 & 77 & 73 & 81 & 82 & 78 & 68 & 72 & 80 & 60 & 71 & 75 & 75 \\
\hline \multicolumn{14}{|c|}{ Radiação solar global diária (MJ m²) } \\
\hline 2001 & 17,72 & 16,99 & 15,61 & 15,42 & 14,56 & 11,51 & 13,58 & 14,48 & 17,60 & 18,66 & 19,91 & 17,16 & 16,10 \\
\hline 2000 & 18,77 & 18,41 & 17,13 & 15,97 & 14,79 & 13,36 & 15,02 & 17,38 & 17,59 & 20,18 & 18,00 & 17,67 & 17,02 \\
\hline \multicolumn{14}{|c|}{ Evaporação do tanque classe "A" (mm) } \\
\hline 2001 & 7,3 & 6,3 & 6,1 & 6,0 & 6,2 & 5,2 & 5,5 & 6,6 & 7,8 & 8,2 & 8,7 & 7,8 & 6,8 \\
\hline 2000 & 6,1 & 5,6 & 5,2 & 5,4 & 5,9 & 5,5 & 6,1 & 7,5 & 8,0 & 9,2 & 6,7 & 5,8 & 6,4 \\
\hline \multicolumn{14}{|c|}{ Insolação (h) } \\
\hline 2001 & 8,8 & 6,5 & 6,9 & 7,8 & 8,2 & 6,1 & 7,3 & 7,2 & 8,5 & 8,8 & 9,4 & 7,5 & 7,8 \\
\hline 2000 & 7,1 & 6,7 & 6,4 & 6,5 & 7,2 & 6,0 & 7,2 & 8,0 & 7,5 & 9,1 & 7,6 & 7,4 & 7,2 \\
\hline \multicolumn{14}{|c|}{ Velocidade do vento $\left(\mathrm{km} \mathrm{d}^{-1}\right)$} \\
\hline 2001 & 165,5 & 135,3 & 115,0 & 129,9 & 129,5 & 191,1 & 205,1 & 266,6 & 248,4 & 231,4 & 205,4 & 246,8 & 189,2 \\
\hline 2000 & 120,1 & 104,2 & 118,8 & 135,6 & 157,4 & 197,6 & 200,0 & 250,3 & 283,2 & 249,2 & 174,0 & 123,0 & 176,1 \\
\hline
\end{tabular}

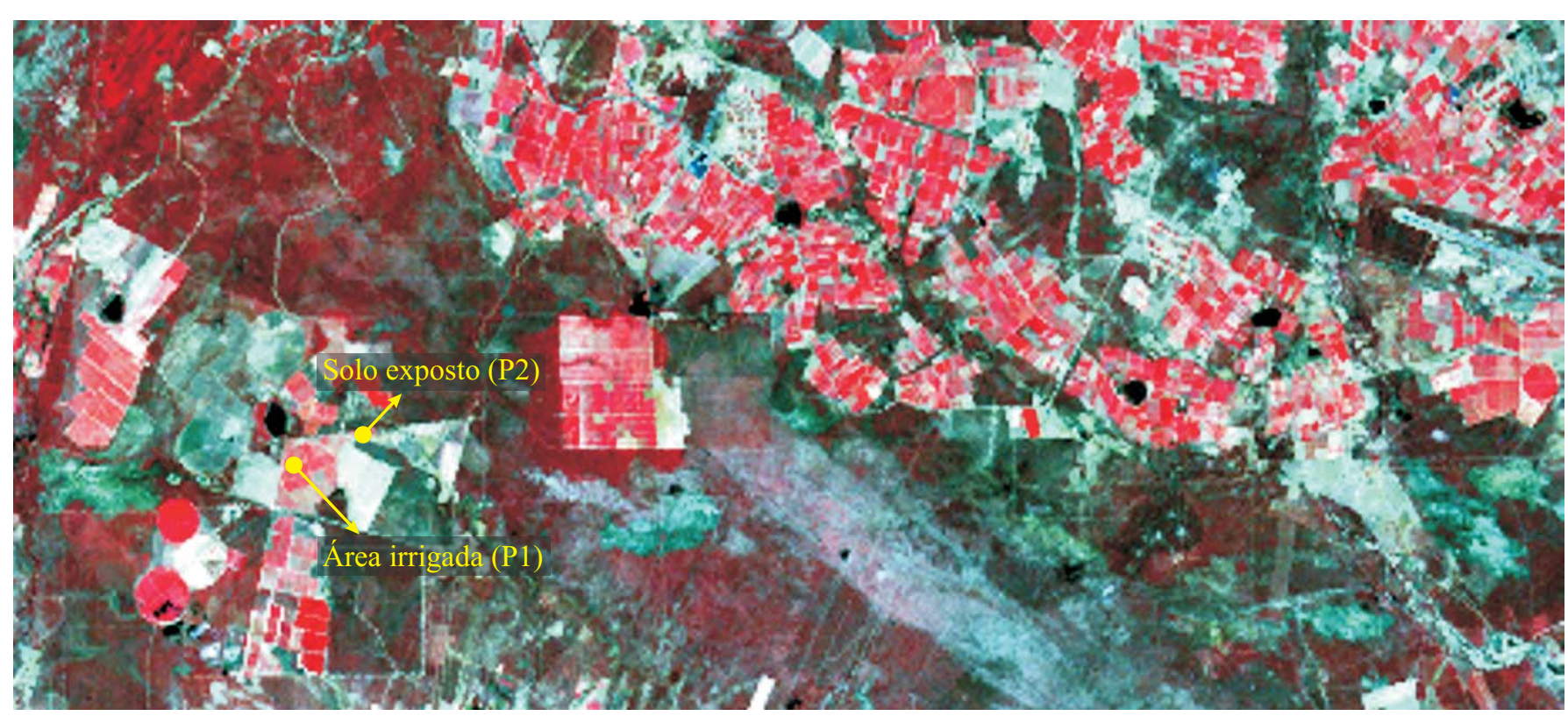

Figura 1. Imagem TM - Landsat 5 em composição falsa cor RGB (red, green and blue) das bandas 2, 3 e 4 referente à área de estudo, com os alvos P1 (área irrigada) e P2 (solo exposto)

cujos valores constam na Tabela 1; ND é a intensidade do pixel (número inteiro compreendido entre 0 e 255 , inclusive) e i corresponde às bandas $(1,2, \ldots$ e 7$)$ do TM Landsat 5.

\section{Reflectância monocromática}

A reflectância monocromática de cada banda $\left(\rho_{\lambda_{\mathrm{i}}}\right)$, definida como sendo a razão entre o fluxo de radiação solar refletido e o fluxo de radiação solar incidente, foi obtida segundo a equação (Allen et al., 2002; Silva et al., 2005):

$$
\rho_{\lambda \mathrm{i}}=\frac{\pi \cdot \mathrm{L}_{\lambda \mathrm{i}}}{\mathrm{k}_{\lambda \mathrm{i}} \cdot \cos Z \cdot \mathrm{d}_{\mathrm{r}}}
$$

em que $\mathrm{L}_{\lambda \mathrm{i}}$ é a radiância espectral de cada banda, $\mathrm{k}_{\lambda_{\mathrm{i}}}$ é a irradiância solar espectral de cada banda no topo da atmosfera $\left(\mathrm{W} \mathrm{m}^{-2} \mu \mathrm{m}^{-1}\right), \mathrm{Z}$ é o ângulo zenital solar e $\mathrm{d}_{\mathrm{r}}$ é o inverso do quadrado da distância relativa Terra-Sol, dada por (Iqbal, 1983): 


$$
\begin{aligned}
\left(\frac{\mathrm{r}}{\mathrm{r}_{\mathrm{o}}}\right)^{2}= & 1,000110+0,034221 \cos \Gamma+0,001280 \operatorname{sen} \Gamma \\
& +0,000719 \cos 2 \Gamma+0,000077 \operatorname{sen} 2 \Gamma
\end{aligned}
$$

em que $\Gamma=2 \pi(\mathrm{D}-1) / 365(\mathrm{rad})$ e $\mathrm{D}$ é a ordem de dia do ano.

\section{Albedo no topo da atmosfera e da superfície}

O albedo no topo da atmosfera $\left(\alpha_{\text {toa }}\right)$, isto é, o albedo nãocorrigido (Eq. 4), expresso pela combinação linear das reflectâncias monocromáticas, e o albedo da superfície $(\alpha)$, corrigido pelos efeitos atmosféricos (Eq. 5), foram obtidos através das equações:

$$
\begin{gathered}
\alpha_{\text {toa }}=0,293 \rho_{1}+0,274 \rho_{2}+0,233 \rho_{3}+0,157 \rho_{4}+0,033 \rho_{5}+0,011 \rho_{7} \\
\alpha=\frac{\alpha_{\text {toa }}-\alpha_{\mathrm{p}}}{\tau_{\text {sw }}^{2}}
\end{gathered}
$$

em que $\rho_{1}, \rho_{2}, \rho_{3}, \rho_{4}, \rho_{5}$ e $\rho_{7}$ são os albedos planetários das bandas $1,2,3,4,5$ e 7 , e $\alpha_{\text {toa }}$ é o albedo planetário, $\alpha_{\mathrm{p}}$ é a radiação solar refletida pela atmosfera, que varia entre 0,025 e 0,04 . No SEBAL, este valor geralmente é considerado igual a 0,03 (Bastiaanssen, 2000; Silva et al., 2005) e $\tau_{\text {sw }}$ é a transmissividade atmosférica, obtida para condições de céu claro em função da altitude de cada pixel, por equação proposta por Allen et al. (2002).

\section{Índices de vegetação: IVDN, IVAS e IAF}

O Índice de Vegetação da Diferença Normalizada (IVDN) foi obtido através da razão entre a diferença das refletividades do infravermelho próximo ( $\left.\rho_{\mathrm{IV}}\right)$ e do vermelho $\left(\rho_{\mathrm{V}}\right)$ e a soma entre elas (Tucker, 1979 apud Tasumi, 2003):

$$
\mathrm{IVDN}=\frac{\rho_{\mathrm{IV}}-\rho_{\mathrm{V}}}{\rho_{\mathrm{IV}}+\rho_{\mathrm{V}}}
$$

em que $\rho_{\mathrm{IV}}$ e $\rho_{\mathrm{V}}$ correspondem, respectivamente, às refletividades das bandas 4 e 3 do TM - Landsat 5. O IVDN é um indicador da quantidade e da condição da vegetação verde da área e seus valores variam de $-1 \mathrm{a}+1$.

Para o cálculo do Índice de Vegetação Ajustado aos Efeitos do Solo (IVAS) utilizou-se a expressão (Huete, 1988):

$$
\mathrm{SAVI}=\frac{(1+\mathrm{L})\left(\rho_{\mathrm{IV}}-\rho_{\mathrm{V}}\right)}{\left(\mathrm{L}+\rho_{\mathrm{IV}}+\rho_{\mathrm{V}}\right)}
$$

em que L é constante, cujo valor mais freqüentemente usado é 0,5 (Accioly et al., 2002; Boegh et al., 2002; Silva et al., 2005).

O Índice de Área Foliar (IAF) é definido pela razão entre a área foliar de toda a vegetação por unidade de área utilizada por essa vegetação; o referido índice é um indicador da biomassa de cada pixel da imagem, sendo computado pela seguinte equação empírica (Allen et al., 2002):

$$
\mathrm{IAF}=-\frac{\ln \left(\frac{0,69-\mathrm{SAVI}}{0,59}\right)}{0,91}
$$

\section{Emissividades}

Como cada pixel não emite radiação eletromagnética como um corpo negro, surge a necessidade de se introduzir a emissividade de cada pixel $\left(\varepsilon_{\mathrm{NB}}\right)$ no domínio espectral da banda termal do TM - Landsat 5 (10,4 a $12,5 \mu \mathrm{m})$; por sua vez, quando do cômputo da radiação de onda longa emitida por cada pixel, deve-se considerar a emissividade de banda larga $\left(\varepsilon_{0}\right)$. Segundo Allen et al. (2002) as emissividades $\varepsilon_{0}$ e $\varepsilon_{\mathrm{NB}}$ podem ser obtidas para IVDN $>0$ e IAF $<3$ com base nas seguintes equações:

$$
\begin{aligned}
& \varepsilon_{\mathrm{NB}}=0,97+0,00331 \mathrm{IAF} \\
& \varepsilon_{0}=0,95+0,01 \mathrm{IAF}
\end{aligned}
$$

Para pixels com IAF $\geq 3, \varepsilon_{\mathrm{NB}}=\varepsilon_{0}=0,98$ e corpos de água (IVDN $<0$ ), $\varepsilon_{\mathrm{NB}}=0,99$ e $\varepsilon_{0}=0,985$ (Allen et al., 2002).

\section{Temperatura da superfície}

A temperatura da superfície $\left(\mathrm{T}_{\mathrm{s}}\right)$, em graus Kelvin, foi determinada em função da radiância espectral da banda 6 (termal) e $\varepsilon_{\mathrm{NB}}$ através da seguinte expressão (Morse et al., 2000; Allen et al., 2002; Silva et al., 2005):

$$
\mathrm{T}_{\mathrm{s}}=\frac{\mathrm{K}_{2}}{\ln \left(\frac{\varepsilon_{\mathrm{NB}} \mathrm{K}_{1}}{\mathrm{~L}_{\mathrm{l}, 6}}+1\right)}
$$

em que $\mathrm{K}_{1}$ e $\mathrm{K}_{2}$ são constantes iguais a $607,76 \mathrm{~W} \mathrm{~m}^{2} \mathrm{sr}^{-1} \mu \mathrm{m}^{-1}$ e $1260,56 \mathrm{~K}$, respectivamente.

\section{Radiação de onda longa emitida e incidente}

A radiação de onda longa emitida por cada pixel $\left(R_{L} \uparrow\right)$ e a radiação de onda longa incidente, emitida pela atmosfera na direção da superfície $\left(\mathrm{R}_{\mathrm{L} \downarrow}\right)$, foram obtidas através das equações:

$$
\begin{aligned}
& \mathrm{R}_{\mathrm{L} \uparrow}=\varepsilon_{0} \sigma \mathrm{T}_{\mathrm{s}}^{4} \\
& \mathrm{R}_{\mathrm{L} \downarrow}=\varepsilon_{\mathrm{a}} \sigma \mathrm{T}_{\mathrm{a}}^{4}
\end{aligned}
$$

em que $\varepsilon_{0}$ é a emissividade de cada pixel, $\sigma$ é a constante de Stefan-Boltzman $\left(\sigma=5,67.10^{-8} \mathrm{~W} \mathrm{~m}^{-2} \mathrm{~K}^{-4}\right), \mathrm{T}_{\mathrm{s}}$ é a temperatura da superfície (K) e $\varepsilon_{\mathrm{a}}$ é a emissividade atmosférica, obtida por: $\varepsilon_{\mathrm{a}}=0,85 .\left(-\ln \tau_{\mathrm{sw}}\right)^{0,99}$ (Bastiaanssen, 1995; Allen et al., 2002).

\section{Radiação de onda curta incidente}

A radiação de onda curta incidente $\left(R_{s} \downarrow\right)$ corresponde ao fluxo de radiação solar (direta e difusa) que atinge a superfície terrestre e que, para condição de céu claro, foi obtida pela expressão (Bastiaanssen, 1995; Morse et al., 2000):

$$
\mathrm{R}_{\mathrm{s} \downarrow}=\mathrm{S} \cos Z \mathrm{~d}_{\mathrm{r}} \cdot \tau_{\mathrm{sw}}
$$

em que $\mathrm{S}$ é a constante solar $\left(1367 \mathrm{~W} \mathrm{~m}^{-2}\right)$, Z é ângulo zenital solar, $d_{r}$ é o inverso do quadrado da distância relativa Terra-Sol e $\tau_{\mathrm{sw}}$ é a transmissividade atmosférica.

Com base nas informações do MED calculou-se o ângulo zenital do $\mathrm{Sol}-\mathrm{Z}_{\mathrm{i}}$ relativo à normal de cada pixel, segundo expressão (Iqbal, 1983): 


$$
\begin{aligned}
\cos Z_{i}= & \operatorname{sen}(\delta)[\operatorname{sen}(\phi) \cos (\delta)-\cos (\phi) \operatorname{sen}(i) \cos (a)] \\
& +\cos (\delta) \cos (i)[\cos (\phi) \cos (i) \\
& +\operatorname{sen}(\phi) \operatorname{sen}(i) \cos (a)] \\
& +\cos (\delta) \operatorname{sen}(i) \operatorname{sen}(a) \operatorname{sen}(h)
\end{aligned}
$$

em que $\delta$ é a declinação do Sol, $\phi$ a latitude do pixel (positiva no Hemisfério Norte e negativa no Hemisfério Sul.), i a inclinação do pixel em relação ao plano do horizonte local, a é o azimute da normal da superfície do pixel e h é o ângulo horário (positivo antes e negativo após o meio-dia).

\section{Saldo de radiação}

$\mathrm{O}$ saldo de radiação à superfície $(\mathrm{Rn})$ foi computado utilizando-se a seguinte equação do balanço de radiação à superfície:

$$
\mathrm{Rn}=\mathrm{R}_{\mathrm{S} \downarrow}-\alpha \mathrm{R}_{\mathrm{S} \downarrow}+\mathrm{R}_{\mathrm{L} \downarrow}-\mathrm{R}_{\mathrm{L} \uparrow}-\left(1-\varepsilon_{\mathrm{o}}\right) \mathrm{R}_{\mathrm{L} \downarrow}
$$

em que $\mathrm{R}_{\mathrm{S} \downarrow}$ é a radiação de onda curta incidente, $\alpha$ é o albedo corrigido de cada pixel, $\mathrm{R}_{\mathrm{L} \downarrow}$ é a radiação de onda longa emitida pela atmosfera na direção de cada pixel, $\mathrm{R}_{\mathrm{L} \uparrow}$ é a radiação de onda longa emitida por cada pixel e $\varepsilon_{\mathrm{o}}$ é a emissividade de cada pixel. As densidades de fluxos $T_{L \uparrow} R_{L \downarrow}, R_{S \downarrow}$ e Rn são expressas em $\mathrm{W} \mathrm{m}^{-2}$.

\section{RESULTADOS E DISCUSSÃO}

As variáveis atmosféricas analisadas com e sem o uso do Modelo de Elevação Digital (MED) nos dias 04/12/2000 e 04/10/2001, são apresentadas na Tabela 3. A emissividade $\left(\varepsilon_{0}\right)$, o índice de vegetação da diferença normalizada (IVDN), o albedo $(\alpha)$, a temperatura da superfície (Ts) e o saldo de radiação $(\mathrm{Rn})$, foram determinados para os alvos $\mathrm{P} 1$ (área irrigada) e P2 (solo exposto). Os valores de $\varepsilon_{0}$ para cada alvo separadamente, foram praticamente iguais nos dias analisados, variando apenas com o tipo de superfície (área irrigada ou solo exposto). No alvo com maior conteúdo de vapor de água (P1) a emissividade foi ligeiramente maior que no alvo com menor conteúdo de água (P2). Na área irrigada a emissividade variou entre 0,99 e 0,98 , enquanto no alvo P2 (solo exposto) ela variou entre 0,971 e 0,974 ; a razão disto reside no fato da absortividade ser maior nas superfícies líquidas que em solo exposto ou com vegetação natural. Esses resultados estão compatíveis com os obtidos por Bastiaanssen (2000) que encontrou emissividade igual a 0,93 em pixel definido como seco e 1,0 para pixel úmido, na Bacia do Lago de Gediz, na Turquia, com a aplicação do SEBAL. Como os valores de emissividade são praticamente constantes para cada um dos alvos analisados e a diferença entre os seus valores típicos é inferior a $1 \%$, esta variável sofre pouca influência da topografia da superfície. Becker \& Li (1990) sugerem que a emissividade da superfície é a causa principal de erros na estimativa de temperatura da superfície com imagens de satélite. Ouaidrari et al. (2002) observaram que a emissividade de áreas vegetadas é próxima a 0,98 , com variação de 0,92 a 0,96, para solos expostos; nas áreas de solo nu esses valores variam entre 0,91 e 0,93.

\begin{tabular}{|c|c|c|c|c|}
\hline \multirow{2}{*}{ Imagem } & \multicolumn{2}{|c|}{ Área irrigada (P1) } & \multicolumn{2}{|c|}{ Solo exposto (P2) } \\
\hline & Com MED & Sem MED & Com MED & Sem MED \\
\hline \multicolumn{5}{|c|}{ Emissividade (adimensional) } \\
\hline $04 / 12 / 2000$ & 0,980 & 0,990 & 0,971 & 0,974 \\
\hline $04 / 10 / 2001$ & 0,980 & 0,980 & 0,974 & 0,974 \\
\hline \multicolumn{5}{|c|}{ IVDN (adimensional) } \\
\hline $04 / 12 / 2000$ & 0,779 & 0,774 & 0,198 & 0,196 \\
\hline $04 / 10 / 2001$ & 0,639 & 0,639 & 0,152 & 0,152 \\
\hline \multicolumn{5}{|c|}{ Albedo (\%) } \\
\hline $04 / 12 / 2000$ & 23,0 & 21,6 & 36,0 & 35,0 \\
\hline $04 / 10 / 2001$ & 18,2 & 18,0 & 39,4 & 36,7 \\
\hline \multicolumn{5}{|c|}{ Temperatura da superficie $\left({ }^{\circ} \mathrm{C}\right)$} \\
\hline $04 / 12 / 2000$ & 24,5 & 24,9 & 31,5 & 33,0 \\
\hline $04 / 10 / 2001$ & 27,1 & 27,1 & 35,9 & 35,9 \\
\hline \multicolumn{5}{|c|}{ Saldo de radiação $(\mathrm{Rn}-\mathrm{W}$ m-2) } \\
\hline $04 / 12 / 2000$ & 543,3 & 583,2 & 431,4 & 434,8 \\
\hline $04 / 10 / 2001$ & 575,2 & 618,0 & 312,6 & 399,5 \\
\hline
\end{tabular}

Tabela 3. Emissividade, índice de vegetação da diferença normalizada (IVDN), albedo $(\%)$, temperatura da superfície $\left({ }^{\circ} \mathrm{C}\right)$ e saldo de radiação (Rn) com e sem MED, para alvos selecionados: P1 (área irrigada) e P2 (solo exposto)

Em 04/12/2000 foram encontrados, no alvo P1, valores instantâneos do IVDN iguais a 0,779 com o MED e de 0,774 sem o MED; já no alvo P2, esses valores foram de 0,198 com o MED e de 0,196 sem o MED. Encontraram-se, no dia 04/ 10/2001, valores do IVDN com e sem MED, exatamente iguais em cada superfície analisada, os quais foram 0,639 e 0,152 em área irrigada e solo exposto, respectivamente; em 04/12/ 2000 ocorreu pequena variação do IVDN (inferior a 1\%) com a aplicação do MED; esses resultados indicam que, praticamente, não ocorre variação no IVDN com e sem o MED em cada alvo analisado. Bastiaanssen (2000) encontrou IVDN em um pixel seco de 0,22 e em pixel úmido de $-0,017$. Silva et al. (2005) obtiveram, em alvo de área cultivada com fruteira, solo exposto e água, valores de IVDN iguais a 0,71, 0,18 e $-0,05$, respectivamente. Huete \& Tucker (1991) constataram que em solo exposto o IVDN varia entre 0,05 e 0,30 .

Os valores do albedo da área irrigada variaram de um mínimo de 18\% (sem MED) ao valor máximo de 23\% (com MED); por outro lado, no solo exposto os valores do albedo foram bastante superiores àqueles do alvo $\mathrm{P} 1$, os quais variaram entre 35 e 39,4\%; portanto, os valores mínimos e máximos do albedo no alvo P2 foram, respectivamente, 41,6 e $48,6 \%$ superiores àqueles observados no alvo P1; porém, nos dois alvos e nos dois anos o albedo foi levemente superior com a aplicação do MED que sem o MED; já na área irrigada os valores do albedo em 2000 e 2001, com o MED, foram, respectivamente, 6,1 e 1,1\% maiores que sem o MED. No solo exposto, esses valores em 2000 e 2001 foram, respectivamente, 2,7 e $6,8 \%$ menores que aqueles obtidos sem o MED.

$\mathrm{O}$ albedo do solo exposto nas duas datas de observação, está dentro da faixa de valores esperados para solos com grande refletividade, em razão da sua pouca capacidade de retenção de umidade. $\mathrm{O}$ solo da área estudada tem textura arenosa com alta capacidade de infiltração e baixa capacidade de retenção de água (Azevedo et al., 2003). Segundo Sellers (1965), o albedo de pomares pode variar de 15 a $20 \%$ e, em 
campos de trigo e arroz, entre 10 a 25\%; o albedo da água varia de 3 a 10\% para ângulo zenital pequeno e de 10 a 50\% para ângulo zenital grande; em solo exposto seco, o albedo varia de 20 a $35 \%$ e, para solo exposto úmido, de 10 a $20 \%$. Chandrapala \& Wimalasuriya (2003) obtiveram, em grandes extensões de dunas de areia na costa Oeste do Sirilanka, albedo entre 34 a $36 \%$. Os autores também constataram que o albedo da superfície do mar, próximo à área continental estudada, foi de 5\%. Kustas et al. (1990), usando técnicas de sensoriamento remoto, obtiveram albedo de 21,23 e $22 \%$ em áreas com trigo, alfafa e algodão, respectivamente.

Nos dois dias de observação, conforme previsto, os valores da temperatura da superfície do solo foram bastante superiores àqueles da temperatura da superfície irrigada. A temperatura da superfície da área irrigada variou entre 24,5 e $27,1^{\circ} \mathrm{C}$ e do solo exposto, entre 31,5 e $35,9^{\circ} \mathrm{C}$; entretanto, com a aplicação do MED esses valores foram iguais no dia 04/10/ 2001 nos dois alvos e, em 04/12/2000, os valores da temperatura da superfície, nos dois alvos analisados, foram apenas $1,6 \%$ maior sem a aplicação do MED que aqueles valores obtidos com a aplicação do MED; este resultado assegura que a aplicação do MED exerce pouca influência no cálculo da temperatura da superfície dos alvos analisados; por outro lado, em 04/10/2001 a temperatura da superfície dos alvos estudados foi aproximadamente $10 \%$ maior que em 04/12/2000; outrossim, os valores de temperatura do ar observados na estação agroclimatológica de Bebedouro, PE, também foram maiores no ano de 2001 que em 2000, tanto nos meses da passagem do satélite quanto na média anual (Tabela 2).

Os valores do saldo de radiação dos dois alvos e nas duas datas analisadas, foram superiores com a aplicação do MED; em 04/12/2000, os valores de Rn da área irrigada com e sem o MED foram, respectivamente, 543,3 e 583,2 $\mathrm{W} \mathrm{m}^{-2}$, enquanto no solo exposto esses valores foram 431,4 e $434,8 \mathrm{~W} \mathrm{~m}^{-2}$, respectivamente, com MED e sem MED; por outro lado, em 04/10/2001 os valores de Rn na área irrigada atingiram 575,2 e $618,0 \mathrm{~W} \mathrm{~m}^{-2}$ com e sem MED, respectivamente, enquanto no solo exposto eles indicaram 312,6 e $399,5 \mathrm{~W} \mathrm{~m}^{-2}$, respectivamente, com MED e sem MED; portanto, a aplicação do MED na área irrigada produziu um decréscimo de aproximadamente $7 \%$ em Rn nas duas datas analisadas, mas no solo exposto com a aplicação do MED esse decréscimo foi muito pequeno no ano de 2000 (0,78\%) e muito grande em 2001 $(21,7 \%)$. Esse resultado evidencia que, quando se considera a inclinação e o aspecto de cada pixel, podem ocorrer variações consideráveis nos valores do saldo de radiação, mesmo em áreas com topografia relativamente plana, como no caso do presente estudo; desta forma, o efeito da utilização do MED dever ser mais acentuado no cálculo do saldo de radiação de bacias hidrográficas, com topografia mais irregular.

Utilizando-se ou não o MED, os valores de Rn do solo exposto calculado com base no satélite Landsat 5 no ano de 2000, foram superiores àqueles de 2001; já na área irrigada esta situação foi inversa. Ressalte-se que a radiação global em Bebedouro foi maior em 04/12/2000 que no dia analisado de 2001 (Tabela 2); este resultado está em concordância com os valores de Rn da área com solo exposto; entretanto, é aparentemente conflitante com os valores obtidos para a área irrigada, cuja razão reside no fato de que o saldo de radiação não é influenciado apenas pelo total de radiação solar global incidente mas, também, e principalmente, pela topografia e tipo de cobertura da superfície. Os valores do saldo de radiação estimados através do SEBAL com a aplicação do MED foram inferiores aos valores indicados com tal modelo sem o MED. Este resultado evidencia a importância de se levar em consideração os efeitos topográficos na estimativa do saldo de radiação, com base em imagens de satélites.

Apresentam-se, nas Figuras 2 e 3, os valores instantâne-
A.

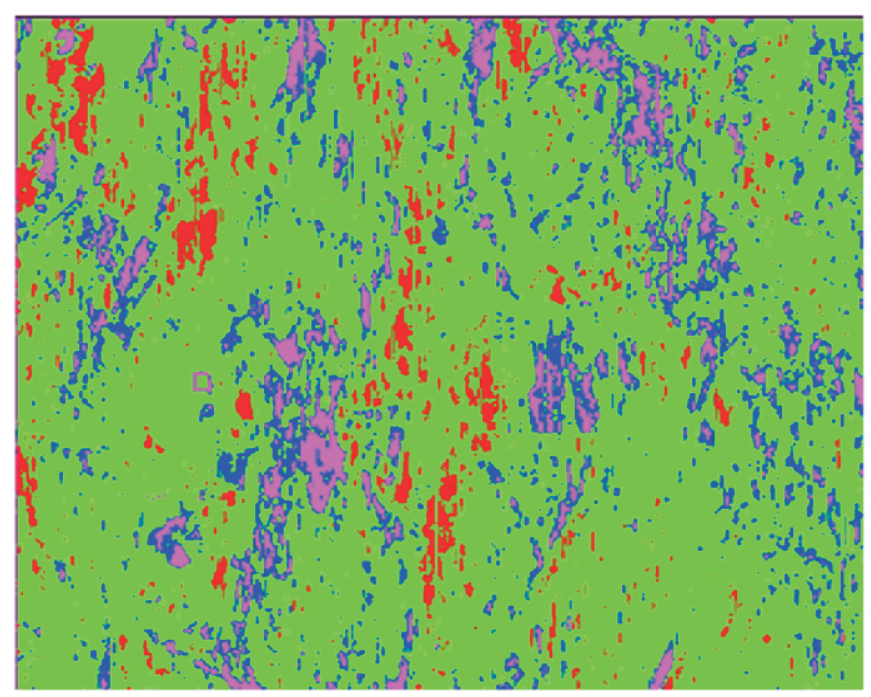

B.

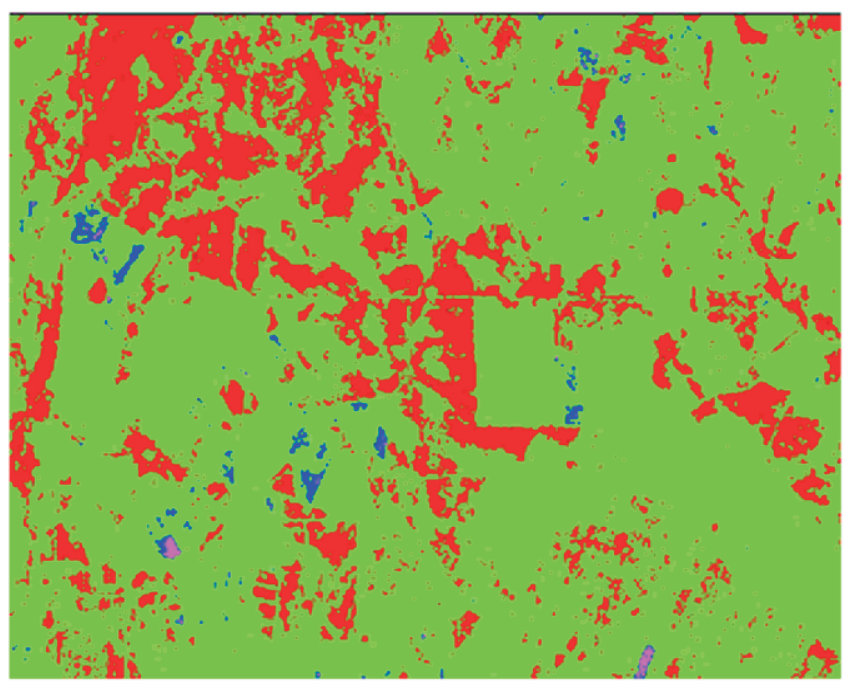

594,9

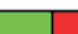

Figura 2. Imagem do saldo de radiação de onda curta (W m²-2) com o MED (A) e sem o MED (B) para o dia 04 de dezembro de 2000 
A.

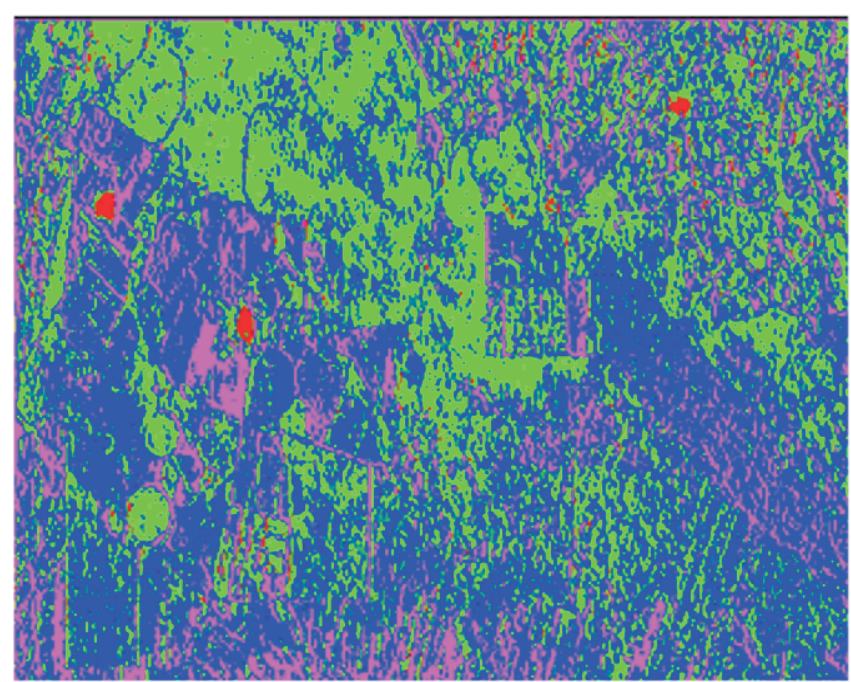

B.

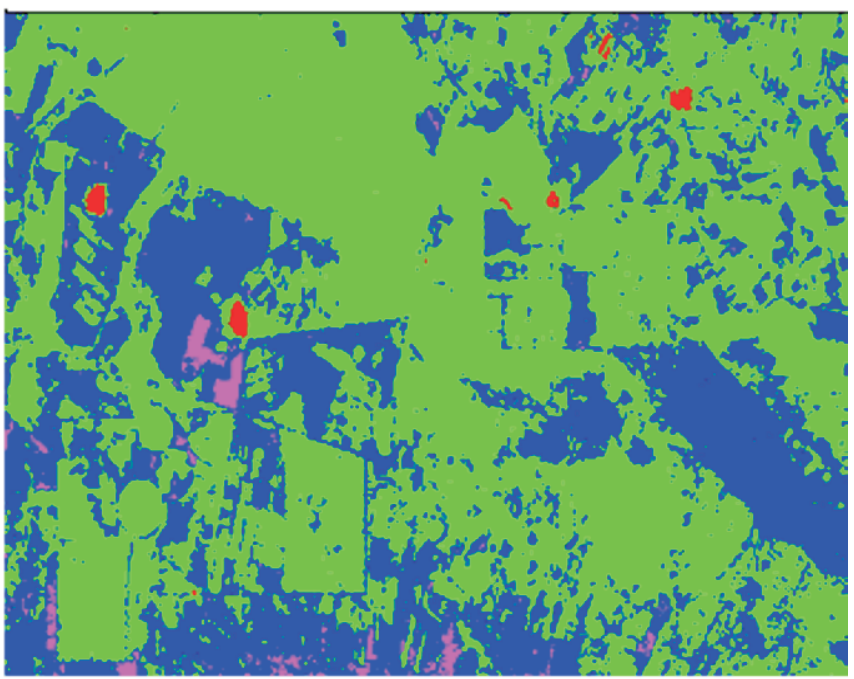

651,9

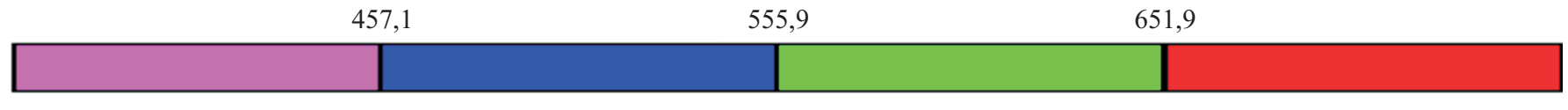

Figura 3. Imagem do saldo de radiação de onda curta $\left(\mathrm{W} \mathrm{m}^{-2}\right)$ com o MED (A) e sem o MED (B) para o dia 04 de outubro de 2001

os do saldo de radiação à superfície da área em estudo, utilizando-se imagens do TM - Landsat 5, dos dias 04 de dezembro de 2000 e 04 de outubro de 2001. Para a imagem do dia 04/12/2000, as áreas com tonalidade rosa representam valores de $\mathrm{Rn}$ inferiores a $360,3 \mathrm{~W} \mathrm{~m}^{-2}$, azul entre 360,3 e $418,9 \mathrm{~W} \mathrm{~m}^{-2}$, verde entre 418,9 e $594,9 \mathrm{~W} \mathrm{~m}^{-2}$ e vermelho superior a $594,9 \mathrm{~W} \mathrm{~m}^{-2}$ (Figura 2); já para a imagem do dia 04/10/2001, as áreas com tonalidade rosa representam valores de $\mathrm{Rn}$ inferiores a $457,1 \mathrm{~W} \mathrm{~m}^{-2}$, azul entre 457,1 e $555,9 \mathrm{~W} \mathrm{~m}^{-2}$, verde entre 555,9 e $651,9 \mathrm{~W} \mathrm{~m}^{-2}$ e vermelho superior a $651,9 \mathrm{~W} \mathrm{~m}^{-2}$ (Figura 3 ).

$\mathrm{O}$ valor da moda de Rn de toda a área estudada em 04/ 12/2000 foi de 503,3 $\mathrm{W} \mathrm{m}^{-2}$ com o MED e de 589,4 $\mathrm{W} \mathrm{m}^{-2}$ sem o MED; para o dia 04/10/2001, obtiveram-se os valores de 550,6 e $579,5 \mathrm{~W} \mathrm{~m}^{-2}$ para a moda com e sem a utilização do MED, respectivamente. Silva (2000), estudando as necessidades hídricas da mangueira na região do Submédio rio São Francisco, obteve o saldo de radiação às 9 h 30 min de 471,8 $\mathrm{W} \mathrm{m}^{-2}$. Teixeira (2000) realizou o balanço de energia durante um ciclo produtivo da bananeira irrigada na mesma região e encontrou valores de Rn às 9 h 30 min próximos a $400,0 \mathrm{~W} \mathrm{~m}^{-2}$. Ávila Netto et al. (2000), em trabalho sobre exigências hídricas da videira também cultivada na mesma região, encontraram valores do $\mathrm{Rn}$ para o dia 21/07/2000 às $9 \mathrm{~h} 30 \mathrm{~min}$, iguais a $331,5 \mathrm{~W} \mathrm{~m}^{-2}$. Bastiaanssen et al. (1998) utilizaram o algoritmo SEBAL para estimar o saldo de radiação sobre a bacia do Rio Heihe (China), em áreas de deserto e de oásis e compararam seus resultados com medidas realizadas na superfície. Eles verificaram que os valores do saldo de radiação estimados pelos dados de satélite foram superiores aos medidos no campo, nas duas áreas estudadas.

\section{CONCLUSÕES}

1. A aplicação do modelo de elevação digital no cálculo da emissividade, IVDN, albedo e temperatura da superfície, é suficiente para produzir variações consideráveis no cálculo do saldo de radiação.

2. Na estimativa do saldo de radiação com base em imagens de satélites, deve-se levar em consideração os efeitos topográficos da região de estudo.

\section{LITERATURA CITADA}

Accioly, L. J.; Pacheco, A.; Costa, T. C. C.; Lopes, O. F.; Oliveira, M. A. J. Relações empíricas entre a estrutura da vegetação e dados do sensor TM/Landsat. Revista Brasileira de Engenharia Agrícola e Ambiental, v.6, n.3, p.492-498, 2002.

Allen, R.; Bastiaanssen, W.; Waters, R.; Tasumi, M.; Trezza, R. Surface energy balance algorithms for land (SEBAL), Idaho implementation - Advanced training and users manual, version 1.0, 2002.97p.

Ávila Netto, J.; Azevedo, P. V.; Silva, B. B. Exigências hídricas da videira na região do sub-médio São Francisco. Pesquisa Agropecuária Brasileira, v.35, n.8, p.1559-1566, 2000.

Azevedo, P. V.; da Silva, B. B.; da Silva, V. P. R. Water requirements of irrigated mango orchards in northeast Brazil. Agricultural Water Management, v.1, n.58, p.241-254, 2003.

Bastiaanssen, W. G. M. Regionalization of surface flux densities and moisture indicators in composite terrain. Wageningen: Wageningen Agricultural University, 1995. 237p. PhD thesis

Bastiaanssen, W. G. M. SEBAL - based sensible and latent heat fluxes in the irrigated Gediz Basin, Turkey. Journal of Hydrology, v.229, p.87-100, 2000. 
Bastiaanssen, W. G. M.; Menenti, M.; Feddes, R. A.; Holtslag, A. A. $\mathrm{M}$. The surface energy balance algorithm for land (SEBAL): Part 1 formulation, Journal of Hydrology, v.212-213, p.198-212, 1998.

Becker, F.; Li, Z. L. Towards a local split window method over land surfaces. International Journal of Remote Sensing, v.11, n.3, p.369-394, 1990.

Boegh, E.; Soegaard, H.; Thomsen, A. Evaluating evapotranspiration rates and surface conditions using Landsat TM to estimate atmospheric resistance and surface resistance. Remote Sensing of Environment, v.79, n.1, p.329-343, 2002.

Chandrapala, L.; Wimalasuiya, M. Satellite measurements supplemented with meteorological data to operationally estimate evaporation in Sri Lanka. Agricultural Water Management, v.58, n.2, p.89-107, 2003.

Granger, R. J. Satellite-derived estimates of evapotranspiration in the Gediz basin. Journal of Hydrology, v.229, p.70-76, 2000.

Huete, A. R. Adjusting vegetation indices for soil influences. International Agrophysics, v.4, n.4, p.367-376, 1988.

Huete, A. R.; Tucker, C. J. Investigation of soil influences in AVHRR red and near - infrared vegetation index ilmagery. International Journal of Remote Sensing, v.12, n.6, p.1223-1242, 1991.

Iqbal, M. An introduction to solar radiation. Library of Congress Cataloging in Publication Data. Canada: Academic Press, 1983. 408p.

Kustas, W. P.; Moran, M. S.; Jackson, R. D.; Gay, L. W.; Duell, L. F. W.; Kunkel, K. E.; Mathias, A. D. Instantaneous and daily values of the surface energy balance over agricultural fields using remote sensing and a reference field in an arid environment. Remote Sensing of Environment, v.32, n.2-3, p.125-141, 1990.
Markham, B. L.; Barker, L. L. Thematic mapper bandpass solar exoatmospherical irradiances. International Journal of Remote Sensing, v.8, n.3, p.517-523, 1987.

Morse, A.; Tasumi, M.; Allen, R. G.; Kramber, W. J. Aplication of the SEBAL methodology for estimating consumptive use of water and streamflow deplition in the Bear River basin of Idaho through remote sensing - Final Report. Boise: Department of Water Resources/University of Idaho, 2000, 98p.

Ouaidrari, H.; Goward, S. N.; Czajkowski, K. P.; Sobrino, J. A.; Vermote, E. Land surface temperature estimation from AVHRR thermal infrared measurements: An assessment for the AVHRR Land Pathfinder II data set. Remote Sensing of Environment, v.81, n.1, p.114-128, 2002.

Sellers, W. D. Physical climatology. Chicago: The Universy of Chicago Press. Chicago \& London, 1965. 272p.

Silva, B. B.; Lopes, G. M.; Azevedo, P. V. Balanço de radiação em áreas irrigadas utilizando imagens Landsat 5 - TM. Revista Brasileira de Meteorologia, v.20, n.2, p.243-252, 2005.

Silva, V. P. R. Estimativa das necessidades hídricas da mangueira. Campina Grande: UFPB, 2000. 129p. Tese Doutorado

Tasumi, M. Progress in operational estimation of regional evapotranspiration using satellite imagery. Idaho: University of Idaho, 2003. 378p. PhD thesis

Teixeira, A. H. C. Balanço de energia na cultura da bananeira no primeiro ciclo de produção. In: Congresso Brasileiro de Meteorologia, 11, 2000, Rio de Janeiro. Anais... Rio de Janeiro: SBA, 2000, CD-Rom 